\title{
An Overview of the Malaria Epidemic in Sub- Saharan Africa
}

\author{
Sandra M. KONJI* 1
}

1 Student, University of Ottawa, Canada

*Auteur(e) correspondant | Corresponding author : skonj029@uottawa.ca

Résumé :

(traduction)

Mots-clés :
La malaria est une maladie parasitaire transmise par les moustiques lorsqu'ils se nourrissent de sang. Le risque de contracter la malaria est le plus élevé chez les personnes qui habitent les pays tropicaux en raison de l'humidité omniprésente qui favorise les infections. En conséquence, le taux de mortalité des femmes et des enfants atteints de malaria est disproportionnellement élevé en Afrique subsaharienne. Le manque d'éducation sanitaire et de connaissances sur la malaria constitue d'être un obstacle important contre l'efficacité de la prévention et du traitement de cette maladie. Le manque d'éducation sanitaire a réduit l'efficacité des agents antimalariques, comme le médicament Artemether Lumefantrine, car ceux-ci sont distribués et administrés par des personnes non formées. En raison du manque de données sur l'incidence et de données de prévalence, il est difficile d'approvisionner adéquatement les pays endémiques en médicaments. De plus, le manque de connaissances sur la pathogenèse et la transmission de la malaria a empêché plusieurs personnes à se faire traiter rapidement et à adopter des mesures préventives. Récemment, la mise en œuvre de programmes d'éducation sanitaire par des organismes internationaux a permis aux professionnels de la santé locaux et ambulants d'être éduqués sur la maladie et sur les méthodes d'administration d'agents antimalariques.

Malaria, Plasmodium falciparum, Afrique subsaharienne, niveau d'instruction, moustique Anopheles

Malaria is a parasitic disease that is transmitted by mosquitos during their blood meal. The risk of contracting malaria is highest for people in tropical countries, due to the ever-present humid weather that allows yearly infections. Consequently, sub-Saharan Africa has a disproportionately higher rate of death among women and children with malaria. One of the major barriers identified in the efficacy of malaria treatment and prevention is the lack of health education and literacy. The lack of health education has decreased the efficacy of antimalarial drugs, such as Artemether Lumefantrine, due to the distribution and administration of the drug by untrained persons. The lack of incidence and prevalence data makes it difficult to ensure adequate supply of the drug in endemic countries. Furthermore, the lack of knowledge of malaria pathogenesis and transmission has prevented many from promptly seeking treatment and practicing preventative care methods. Recently, the implementation of health education programs by international organizations has allowed local and travelling healthcare practitioners to be educated on the disease and methods of antimalarial drug administration.

Malaria, Plasmodium falciparum, sub-Saharan Africa, education and literacy,
Anopheles mosquito
Keywords: 


\section{Introduction}

Malaria is a disease caused by a parasitic protozoan of the genus Plasmodim that can live in humans and mosquitoes, specifically female Anopheles mosquitoes (Sinclair, Zani, Donegan, Olliaro, \& Garner, 2009). There are four different species of Plasmodium that cause malaria in humans and they are $P$. falciparum, $P$. malariae, $P$. ovale, and $P$. vivax (Sinclair et al., 2009). In 2013, 97 countries in Africa, South East Asia, and South America had ongoing malaria transmission (World Health Organization [WHO], 2011). According to the WHO (2011), an estimated 3.4 billion people are at risk of malaria. Ninety percent of all malaria deaths occur in sub-Saharan Africa, where the most prevalent malaria parasite is P. falciparum (White et al., 2014). In 2012, 500 ooo children under five years of age died this translates to 1300 children per day (WHO, 2011).

\section{Life Cycle and Pathogenesis of Plasmodium Falciparum}

An infected female Anopheles mosquito carries male and female gametocytes that contain malaria (White et al., 2014). The gametocytes develop into oocysts, which mature and release sporozoites into the salivary glands of the mosquito. The Anopheles mosquito bites humans because the protein and iron found in blood nourishes their eggs. While the mosquito draws blood, the sporozoites that were in the salivary glands are injected into the human. If another mosquito bites the same human, it will ingest the blood and sporozoites. The injected sporozoites will then travel to the liver and invade the mosquito's liver cells. In the liver, the sporozoites mature into merozoites. The merozoites transform into the male (micro) and female (macro) gametocytes that are re-injected into the human while feeding.

As a result, the malaria cycle is perpetuated by the repeated transmission of gametocytes and ingestion of sporozoites while the mosquito feeds on blood (Augustincic et al., 2015).

The erythrocytic cycle of the parasite is responsible for the clinical manifestation of malaria symptoms: recurring fevers and chills (Kone et al., 2013). The pathogenic process of malaria is characterized by the bursting of erythrocytecontaining merozoites (White et al., 2014). The merozoites perpetuate reinfection by invading other erythrocytes and releasing adhesive proteins that increase parasitic numbers in the blood. If left untreated, malaria can lead to severe anaemia due to the destruction of erythrocytes, as well as respiratory distress due to low oxygen delivery by erythrocytes (Kone et al., 2013). Additionally, aggregation of erythrocytes to the endothelium can cause cerebral lesions that can lead to depression, impaired memory, and personality changes (White et al., 2014). Finally, the peptides on the parasite have low immunogenicity response, which can inhibit their destruction by antibodies. As a result, those that are infected can develop partial immunity with asymptomatic infections.

$P$. falciparum malaria is very hard to control in subSaharan Africa due to year-round infection opportunities (Augustincic et al., 2015). Frequent epidemics are common because of changes in the environment, a lack of education, and an absence of resources to fund treatment. This paper will analyze the relationship between health education and literacy, and the efficacy of treatment of $P$. falciparum in sub-Saharan Africa.

\section{Discussion}

Studies have shown a positive correlation between health education and the efficacy of treatment (Kroeger, Meyer, Mancheno, \& Gonzalez, 1996). During 1970s and the 1980 , malaria was reasonably well controlled due to high awareness among the population and the use of intermittent preventative treatment (IPT) practices (antenatal administration of sulfadoxine-pyrimethamine drug and mosquito nets) to treat and prevent malaria among children (Paul et al., 2015). However, the spread of drug resistance and a poor understanding of the disease have led to adverse consequences for malaria control. For example, a study conducted on East African mothers reported that many believe that IPT of malaria during pregnancy weakens the mother and causes poor birth outcomes. In fact, $77 \%$ of mothers believed that malaria is not preventable. This misconception can negatively affect the likelihood of mothers participating in preventative behaviour to protect their children from the disease. Moreover, the false assumption that signs and symptoms of malaria will dissipate on their own discourages residents from seeking treatment (Staedke et al., 2009). In fact, studies have shown that only $60 \%$ of fevers caused by malaria seek treatment, and only one third of those cases strictly adhere to the treatment as to clear parasitaemia (Galactionova et al., 2015). Consequently, more than half of the potential benefits of treatment are lost due to insufficient patient compliance, which can be attributed to a lack of proper education.

The lack of health literacy on malaria medication has decreased the efficacy of available drugs used in the treatment of malaria (Sinclair et al., 2009). To better serve rural inhabitants, many small communities have created makeshift dispensaries on the side of rural roads (Opiyo, Yamey, \& Garner, 2016). However, many of these vendors have not received adequate training on the administration of the medication, such as the required dosage, the drug sched- 
ule, the requirement to ingest the drug with fatty foods, and instructions on re-administration (Maxmen, 2012).

In countries where malaria is endemic, the lack of federal training guidelines for public health educators has significantly limited the treatment and control of the disease (Opiyo et al., 2016). For example, patients suspected of having uncomplicated P. falciparum malaria are treated with Artemether Lumefantrine, a common treatment that uses Artemisinin combination therapy (ACT) to provide rapid relief of malaria symptoms in children (Sinclair et al., 2009). This therapy can clear $100 \%$ of the parasite if given within the first 24 hours of the onset of symptoms (Opiyo et al., 2016). The administration of this therapy would only cost 6-12 USD per person, provided that the dosage and administration of the drug is correctly implemented (Goodman, Coleman, \& Mills, 1999). However, the implementation of ACT therapy as a method of treatment was greatly delayed in some sub-Saharan African countries due to a bottleneck in funding to train healthcare practitioners (Goodman et al., 1999). As a result, there has been noncompliance with national guidelines on the proper administration of ACT as a malaria treatment (Sinclair et al., 2009). Consequently this has led to over-prescription of the drug, and has more than doubled the costs of ACT therapy to 1629 USD per patient (Staedke et al., 2009).

The lack of prevalence and incidence data in the rural areas of sub-Saharan Africa makes it difficult to forecast the disease (Opiyo et al., 2016). Because many residents are reluctant to seek treatment when exhibiting malaria symptoms, they often self-prescribe antimalarial drugs, which makes tracking disease prevalence by drug purchase and collecting incidence data problematic (Kone et al., 2013). The inability to forecast the disease can make it difficult to predict regional demands of antimalarial drugs, thus creating a shortage of the medication (Opiyo et al., 2016). Moreover, antimalarial drugs expire within six months of the manufacturing date, meaning they cannot be stockpiled (Sinclair et al., 2009). As a result, there could be a surplus of drugs in areas with lower malaria incidence and a shortage of the medication in areas with a greater malaria incidence (Maxmen, 2012). All of these factors combined result in large inefficiencies of malaria service and control in endemic countries.

Recently, non-governmental organizations have stepped in to provide health education and preventative tools in countries where malaria is endemic (Opiyo et al., 2016). The implementation of an antimalarial treatment policy by the WHO has helped hospitals and clinics to refine treatment protocols through adequate training of staff and community workers (WHO, 2011). Education of expectant mothers has helped to decrease many of the misconceptions surrounding the use of IPT, thus reducing the frequency of malaria infections among pregnant women and children younger than five years of age (Amoran, 2013). Federal governments have also invested in commercials to educate the public on malaria transmission as well as methods of protection (Augustincic et al., 2015). By increasing health education and literacy on malaria, as many as 274 million cases and 1.1 million deaths in sub-Saharan Africa have been prevented (Kroeger et al., 1996).

\section{Conclusion}

$P$. falciparum is a mosquito-transmitted infectious disease and a large proportion of the disease burden is carried by sub-Saharan Africa. The disease has a higher prevalence among those that are immunocompromised, often including children and pregnant women. One of the largest barriers inhibiting malaria treatment is a lack of education and literacy, which has caused misconceptions about disease development and prevention (Kroeger et al., 1996). Consequently, there has been overuse and misuse of malaria drugs due to self-diagnosis and self-prescription, a practice that has rendered cheaper drugs such as chloroquine and sulphadoxine-pyrimethamine ineffective (Opiyo et al., 2016).

To combat malaria, preventative methods must be targeted towards the vulnerable population. First, local communities must receive direct support in the form of sufficient training for practitioners on accurate diagnosis and medical administration of anti-malarial drugs. Second, the formation of a mobile team of practitioners could educate rural residents on disease transmission as well as methods of prevention. Third, tracking local antimalarial drug purchases would assist in determining the prevalence of the disease. Another future endeavour would be to direct more research and funding efforts towards vaccine development. The implementation of these steps would go a long way in controlling the disease in endemic areas.

\section{References}

Amoran, O. E. (2013). Impact of health education intervention on malaria prevention practices among nursing mothers in rural communities in Nigeria. Nigerian Medical Journal, 54(2), 115-122. doi:10.4103/0300-1652.110046

Augustincic, L. P., Petkovic, J., Welch, V., Ueffing, E., Ghogomu, E. T., Pardo, J. P.,...Tugwell, P. (2015). Strate- 
gies to increase the ownership and use of insecticidetreated bednets to prevent malaria. Cochrane Database of Systemic Reviews, 3, CDoog186.

doi:10.1002/14651858.CDoo9186. pub2.

Galactionova, K., Tediosi, F., de Savigny, D., Smith, T., \& Tanner, M. (2015). Effective coverage and systems effectiveness for malaria case management in sub-Saharan African countries. PLoS One, 1O(5), e0127818. doi:10.1371/ journal.pone.0127818

Goodman, C. A., Coleman, P. G., \& Mills, A. J. (1999). Costeffectiveness of malaria control in sub-Saharan Africa. Lancet, 354(9176), 378-385. doi:10.1016/So140-6736(99)02141 $-8$

Kone, G., Lalou, R., Audibert, M., Lafarge, H., Dos Santos, S., \& Le Hesran, J.-I. (2013). Use of health care among the urban poor in Africa: Does the neighbourhood have an impact? Retrieved from https://halshs.archivesouvertes.fr/halshs-00878946

Kroeger, A., Meyer, R., Mancheno, M., \& Gonzalez, M. (1996). Health education for community-based malaria control: An intervention study in Ecuador, Colombia and Nicaragua. Tropical Medicine \& International Health, 1(6), 836-846.

Maxmen, A. (2012). Public health: Death at the doorstep. Nature, 484(7395), S19-S21. doi:10.1038/484S19a

Opiyo, N., Yamey, G., \& Garner, P. (2016). Subsidising artemisinin-based combination therapy in the private retail sector. Cochrane Database of Systematic Reviews.

doi:10.1002/14651858.CDoo9926.pub2

Paul, C., Kramer, R., Lesser, A., Mutero, C., Miranda, M. L., \& Dickinson, K. (2015). Identifying barriers in the malaria control policymaking process in East Africa: Insights from stakeholders and a structured literature review. BMC Public Health, 15(1), 862-869. doi:10.1186/s12889-015-2183-6

Sinclair, D., Zani, B., Donegan, S., Olliaro, P., \& Garner, P. (2009). Artemisinin-based combination therapy for treating uncomplicated malaria. Cochrane Database of Systematic Reviews. doi:10.1002/14651858.CDoo7483.pub2

Staedke, S. G., Mwebaza, N., Kamya, M. R., Clark, T. D., Dorsey, G., Rosenthal, P. J., \& Whitty, C. J. (2009). Home management of malaria with artemether-lumefantrine compared with standard care in urban Ugandan children: A randomised controlled trial. Lancet, 373(9675), 1623-1631. doi:10.1016/So140-6736(o9)60328-7
White, N. J., Pukrittayakamee, S., Hien, T. T., Faiz, M. A., Mokuolu, O. A., \& Dondorp, A. M. (2014). Malaria. Lancet, 383(9918), 723-735. doi:10.1016/So140-6736(13)60024-o

World Health Organization. (2011). Goals, targets, policies and strategies for malaria control and elimination. In World Malaria Report, 2011(2). Retrieved from http:// www.who.int/malaria/world_malaria_report_2011/ WMR2011_chapter2.pdf 